\title{
Deep search for companions to probable young brown dwarfs ${ }^{\star}$ VLT/NACO adaptive optics imaging using IR wavefront sensing (Research Note)
}

\author{
G. Chauvin ${ }^{1,2}$, J. Faherty ${ }^{3,4}$, A. Boccaletti ${ }^{5}$, K. Cruz $^{4,6}$, A.-M. Lagrange ${ }^{1}$, B. Zuckerman ${ }^{7}$, M. S. Bessell ${ }^{8}$, J.-L. Beuzit ${ }^{1}$,
} M. Bonnefoy ${ }^{2}$, C. Dumas ${ }^{9}$, P. Lowrance ${ }^{10}$, D. Mouillet ${ }^{1}$, and I. Song ${ }^{11}$

${ }^{1}$ UJF-Grenoble1/CNRS-INSU, Institut de Planétologie et d'Astrophysique de Grenoble UMR 5274, 38041 Grenoble, France e-mail: Gael.Chauvin@obs.ujf-grenobla.fr

2 Max Planck Institute for Astronomy, Königstuhl 17, 69117 Heidelberg, Germany

3 Departmento de Astronomia, Universidad de Chile, Casilla 36-D, Santiago, Chile

${ }^{4}$ Department of Astrophysics, American Museum of Natural History, Central Park West at 79th Street, New York, NY 10024, USA

5 LESIA-Observatoire de Paris, CNRS, UPMC Univ. Paris 06, Univ. Paris-Diderot, 92195 Meudon, France

6 Department of Physics \& Astronomy, Hunter College, City University of New York, 695 Park Avenue, New York, NY 10065, USA

${ }^{7}$ Department of Physics \& Astronomy and Center for Astrobiology, University of California: Los Angeles, Box 951562, CA 90095, USA

${ }^{8}$ Research School of Astronomy and Astrophysics Institute of Advance Studies, Australian National University: Cotter Road, Weston Creek, Canberra, ACT 2611, Australia

9 European Southern Observatory, Casilla 19001, Santiago 19, Chile

10 Infrared Processing and Analysis Center, MS 100-22, California Institute of Technology, Pasadena, CA 91125, USA

11 Department of Physics \& Astronomy, University of Georgia, Athens, GA 30602-2451, USA

Received 19 April 2012 / Accepted 14 August 2012

\begin{abstract}
Aims. We have obtained high contrast images of four nearby, faint, and very low mass objects 2MASS J043514551414468, SDSS J044337.61+000205.1, 2MASS J06085283-2753583 and 2MASS J06524851-5741376 (hereafter 2MASS0435-14, SDSS0443+00, 2MASS0608-27 and 2MASS0652-57), identified in the field as probable isolated young brown dwarfs. Our goal was to search for binary companions down to the planetary mass regime.

Methods. We used the NAOS-CONICA adaptive optics instrument (NACO) and its unique capability to sense the wavefront in the near-infrared to acquire sharp images of the four systems in $K_{\mathrm{s}}$, with a field of view of $28^{\prime \prime} \times 28^{\prime \prime}$. Additional $J$ and $L^{\prime}$ imaging and follow-up observations at a second epoch were obtained for 2MASS0652-57.

Results. With a typical contrast $\Delta K_{\mathrm{s}}=4.0-7.0 \mathrm{mag}$, our observations are sensitive down to the planetary mass regime considering a minimum age of 10 to $120 \mathrm{Myr}$ for these systems. No additional point sources are detected in the environment of 2MASS0435-14, SDSS0443+00 and 2MASS0608-27 between 0.1-12" (i.e. about 2 to $250 \mathrm{AU}$ at $20 \mathrm{pc}$ ). 2MASS0652-57 is resolved as a $\sim 230$ mas binary. Follow-up observations reject a background contaminate, resolve the orbital motion of the pair, and confirm with high confidence that the system is physically bound. The $J, K_{\mathrm{s}}$ and $L^{\prime}$ photometry suggest a $q \sim 0.7-0.8$ mass ratio binary with a probable semi-major axis of 5-6 AU. Among the four systems, 2MASS0652-57 is probably the less constrained in terms of age determination. Further analysis would be necessary to confirm its youth. It would then be interesting to determine its orbital and physical properties to derive the system's dynamical mass and to test evolutionary model predictions.
\end{abstract}

Key words. brown dwarfs - binaries: close - instrumentation: adaptive optics - techniques: image processing

\section{Introduction}

The statistical properties of low-mass star and brown dwarf multiples set stringent constraints on star-formation theories (see Duchene et al. 2007, for a review). Multiplicity frequency, mass ratio and separation distributions can be compared between starforming regions (SFRs) of various ages and densities and the older field population. Direct imaging surveys of very low mass objects in the field yield a binary frequency of $20-30 \%$ for M dwarfs (Marchal et al. 2003; Janson et al. 2012) and 15\%

* Based on observations collected at the European Southern Observatory, Chile (ESO programmes 076.C-0554(A), 076.C-0554(B) and 085.C-0257(A). for L and T dwarfs (e.g., Bouy et al. 2003; Burgasser et al. 2003). Among young systems, Ahmic et al. (2007) and Biller et al. (2011) derive a binary fraction of less than $11 \%$ and $9 \%$ in the Chamaeleon I and the Upper Sco regions respectively. They both confirm the trend observed in the field of a massdependency of the binary frequency. A higher multiplicity rate in SFRs compared with the field, as for T Tauri stars, is not seen for young late-type $M$ dwarfs. However the multiplicity properties are likely to be different as evidenced by the discovery of a population of wide ( $>15 \mathrm{AU})$ brown dwarf binaries in young, nearby clusters (Chauvin et al. 2004; Jayawardhana \& Ivanov 2006; Béjar et al. 2008; Todorov et al. 2010). Individual, young, and tight binaries are particularly important for a direct 
Table 1. Description of the target properties.

\begin{tabular}{|c|c|c|c|c|c|c|c|c|c|c|}
\hline Name & $\begin{array}{c}\alpha \\
{[\mathrm{J} 2000]}\end{array}$ & $\begin{array}{c}\delta \\
{[\mathrm{J} 2000]}\end{array}$ & $\begin{array}{c}\mu_{\alpha}{ }^{a} \\
\text { (mas/yr) }\end{array}$ & $\begin{array}{c}\mu_{\delta}^{a} \\
\text { (mas/yr) }\end{array}$ & $\begin{array}{c}V_{\mathrm{rad}} \\
\left(\mathrm{km} \mathrm{s}^{-1}\right)\end{array}$ & SpT & $\begin{array}{c}d^{a} \\
(\mathrm{pc})\end{array}$ & $\begin{array}{c}J \\
(\mathrm{mag})\end{array}$ & $\begin{array}{c}K \\
(\mathrm{mag})\end{array}$ & Ref. $^{b}$ \\
\hline 2MASS0435-14 & 043514.6 & -141447 & $9 \pm 14$ & $16 \pm 14$ & & $\operatorname{M} 6 \delta \pm 1$ & $8.6 \pm 1.0$ & 11.88 & 9.95 & 1 \\
\hline SDSS0443+00 & 044337.6 & +000205 & $28 \pm 14$ & $-99 \pm 14$ & & M9 $\gamma$ & $16.2 \pm 2.1$ & 12.51 & 11.22 & $1,2,3,4$ \\
\hline 2MASS0608-27 & 060852.8 & -275358 & $8.9 \pm 3.5$ & $10.7 \pm 3.5$ & $24 \pm 1$ & 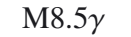 & $31.2_{-32}^{+4.0}$ & 13.59 & 12.37 & $1,5,6$ \\
\hline 2MASS0652-57 & 065248.5 & -574138 & $0.1 \pm 3.4$ & $29.2 \pm 3.3$ & & $\mathrm{M} 8 \beta$ & $31.9_{-2.9}^{+3.7}$ & 13.63 & 12.45 & 6,7 \\
\hline
\end{tabular}

Notes. ${ }^{(a)}$ Distances derived from spectrophotometry with $M_{J}$ estimated from the spectral type/ $M_{J}$ calibration from Cruz et al. (2003) for 2MASS0435-14 and SDSS0443+00. Proper motion and parallax measurements from Faherty et al. (2012) for 2MASS0608-27 and 2MASS065257. ${ }^{(b)}$ References: (1) Cruz et al. (2003), (2) Cruz et al. (2007), (3) Reid et al. (2008), (4) Reiner \& Basri (2009), (5) Rice et al. (2010), (6) Faherty et al. (2012), and (7) Reid et al. (2008).

determination of the dynamical mass to calibrate theoretical masses derived from evolutionary models (Mathieu et al. 2007; Bonnefoy et al. 2009).

For very low mass binaries with high mass ratio, the mass of the binary companion can enter the planetary mass regime. The origin of such a population of planetary mass companions (PMCs) can be hard to infer as the stellar and planetary formation mechanisms probably overlap. From radial velocity surveys, the observed frequency of giant planets around $\mathrm{M}$ dwarfs at small separation $(<3 \mathrm{AU})$ is relatively small $(f<1-2 \%$; Bonfils et al. 2012) compared with solar-type stars $(f<6-9 \%$; Udry $\&$ Santos 2007) and could indicate a mass dependency of the core accretion mechanism efficiency to form giant planets. At larger separations, the situation is less clear. We may expect that alternative mechanisms to core accretion such as cloud or disk fragmentation may form a population of planetary mass companions such as 2M1207 b (Chauvin et al. 2004). Consequently, despite the fact that most exoplanet imaging surveys are now biased towards young, intermediate mass stars, the search for PMCs around low-mass stars and brown dwarfs remains important to understand how planetary formation evolves with the stellar mass and the distance to the star (Delorme et al. 2012).

In the course of our deep imaging survey of 88 young, nearby stars with NACO at VLT (Chauvin et al. 2010), an additional sub-sample of four probable intermediate-young brown dwarfs were observed taking advantage of the infrared (IR) wavefront sensing system of the NACO adaptive optics (AO) instrument. One is a recently confirmed member of the $\beta$ Pictoris moving group (Rice et al. 2010) while the other 3 are low-gravity M dwarfs indicating a likely age lower than a few hundred Myr. We report, in Sect. 2, a summary of the target properties. In Sect. 3, we describe our observations, including the instrument setup and the atmospheric conditions. In Sect. 4, we present the results of this imaging campaign, in terms of detection limits. We also report that 2MASS0652-57 is a $\sim 230$ mas binary. Observations at 2 epochs enable us to resolve the system orbital motion. Finally, we briefly discuss the status of this young binary, and of its membership to any known young moving groups.

\section{Target properties}

2MASS0435-14, SDSS0443+00, 2MASS0652-57, and 2MASS0608-27 were selected as imaging candidates because they have spectral or photometric features indicative of youth (as described below). To date, only 2MASS0608-27 has been confirmed as a $\beta$ Pictoris moving group member. Their properties are summarized in Table 1. As suggested by Kirkpatrick (2005) and further discussed by Kirkpatrick et al. (2006), we indicate spectra with low gravity indicators with greek suffixes: subtle low-gravity features (Pleiades-like) are indicated with a $\beta$, prominent low-gravity features (TWA-like) are indicated with a $\gamma$, while very young (Taurus-like) objects are indicated with a $\delta$. An $\alpha$ suffix is implied for normal-gravity objects. We used these guidelines to assign spectral types to these young/low-gravity $M$ dwarfs even though the low-gravity $\mathrm{M}$ dwarf spectral-typing scheme has yet to be formalized.

- 2MASS0435-14: this object was reported as a young object by Cruz et al. (2003; see Table 11 and Fig. 10) based on spectral features similar to young objects: weak $\mathrm{CaH}$ and $\mathrm{K} \mathrm{I}$ doublet absorption and $\mathrm{NaI}$ and $\mathrm{H}_{\alpha}$ emission. It is flagged as a photometric and kinematic outlier in Faherty et al. (2009) due to its unusual red near-infrared color and very small tangential velocity $\left(v_{\tan }=1 \pm 1 \mathrm{~km} \mathrm{~s}^{-1}\right)$. The low-gravity spectral features of 2MASS0435-14 are similar to those seen in members of very young regions like Taurus. Visual inspection of the 2MASS and IRAS images reveals a significant amount of extinction in the vicinity of the object and it is near the MBM 20/LDN 1642 molecular cloud. Taking reddening into account, this object's red color is likely due to this material rather than condensates present in the object's atmosphere. The spectrum most resembles CHSM1982, an M6 object in Chameleon (Luhman 2004), and thus we tentatively assign a spectral type of M6 $\delta \pm 1$. This object warrants further investigation as it is likely a very young, very low mass object that appears to be isolated in a molecular cloud. - SDSS0443+00: intially classified as a field, old late-M dwarf by Cruz et al. (2003), this object was re-classified by Cruz et al. (2007; see Table 8 and Fig. 5) as it displays low gravity features similar to those seen in late-M type TWA members. Its optical spectrum is similar to Roque 4, an M9 Pleiad (Zapatero Osorio et al. 1998), but seems to have a lower gravity. We therefore assign a spectra type of M9 $\gamma$.

- 2MASS0608-27: this object was first identified by Cruz et al. (2003) as a low-gravity and young brown dwarf candidate based on enhanced spectral VO absorption, as well as weak $\mathrm{CaH}$ and $\mathrm{KI}$ doublet absorption compared with older field dwarfs. More recently, a detailed study of its near-infrared spectral features (highly peaked $H$-band shape, intermediate widths and depth of the gravity-sensitive KI lines) and kinematics and space location, unambiguousy confirmed it as the latest-type non companion member of the $\beta$ Pictoris group. Considering an age of $12 \mathrm{Myr}$ and an assigned spec-

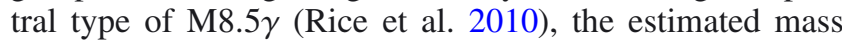
of $15-20 M_{\text {Jup }}$.

- 2MASS0652-57: for this system, suspicion of youth was based on a low-resolution optical spectrum that shows hints of low-gravity, including enhanced spectral VO absorption and weak $\mathrm{NaI}$ and $\mathrm{CaH}$ absorptions. The low-gravity 
Table 2. Detail of the observing parameters.

\begin{tabular}{lcccccccc}
\hline \hline Name & UT date & Filter/obs & $\begin{array}{c}\text { DIT } \\
(\mathrm{s})\end{array}$ & NDIT & NINT & Airmass & $\begin{array}{c}\text { Strehl } \\
(\%)\end{array}$ & $\begin{array}{c}F W H M \\
(\mathrm{mas})\end{array}$ \\
\hline SDSS0443+00 & $2006-08-01$ & $K_{\mathrm{s}} / \mathrm{S} 27$ & 30. & 1 & 11 & 1.29 & 10 & 94 \\
& $2006-02-27$ & $K_{\mathrm{s}} / \mathrm{S} 27$ & 60. & 1 & 3 & 1.27 & 5 & 130 \\
2MASS0652-57 & $2006-08-01$ & $K_{\mathrm{s}} / \mathrm{S} 27$ & 30. & 2 & 5 & 1.28 & 9 & 95 \\
& & $J / \mathrm{S} 27$ & 30. & 2 & 5 & 1.31 & $<5$ & 200 \\
& & $L^{\prime} / \mathrm{L} 27$ & 0.175 & 100 & 21 & 1.25 & 15 & 118 \\
& $2010-08-21$ & $K_{\mathrm{s}} / \mathrm{S} 27$ & 30. & 2 & 10 & 1.62 & 12 & 90 \\
2MASS0608-27 & $2006-02-27$ & $K_{\mathrm{s}} / \mathrm{S} 27$ & 60. & 1 & 10 & 1.03 & 5 & 130 \\
2MASS0435-14 & $2006-02-27$ & $K_{\mathrm{s}} / \mathrm{S} 27$ & 30. & 2 & 20 & 1.12 & 10 & 95 \\
\hline
\end{tabular}

Notes. DIT and NDIT are the integration time and the number of integrations, respectively.

features are similar to those seen in late-M type Pleiades members, and indeed its optical spectrum looks very similar to Roque 7, an M8-type Pleiad (Martín et al. 2000). We assign a spectra type of M $8 \beta$.

\section{Observations and data reduction}

On January 8th and February 27th 2006, the four brown dwarf candidates were imaged with the NACO instrument of the VLTUT4 (see instrument description in Chauvin et al. 2010). Here we used the unique capability offered by NACO at the VLT to sense the wavefront in the near-infrared with the N90C10 dichroic (90\% of the flux transmitted to the wavefront sensor and $10 \%$ to CONICA). This mode is dedicated to the sharp imaging of very red sources $V-K \geq 6$ (M5 or later spectral type). All sources were imaged in $K_{\mathrm{S}}$ bands (without saturation) in average seeing conditions of $0.8^{\prime \prime}$. 2MASS0652-57, which was resolved as a close binary, was also imaged in $J$ and $L^{\prime}$-bands. The corresponding Strehl ratios, FWHM (full width at half maximum of the diffraction limited point spread function) and other observing parameters in each band are given in Table 2. The AO IR sensing allowed us to close the adaptive optics loop on all sources. In Fig. 1, we have reported respectively all final images.

Classical cosmetic reduction including bad pixels removal, flat-fielding, sky substraction and shift-and-add, was made with the Eclipse ${ }^{1}$ reduction software developed by Devillar (1997). Median filtering by a kernel of $3 \times 3$ pixels was applied to correct for remaining hot pixels. Finally, a high-pass filtering to remove spatial frequencies higher than $3 \times$ FWHM was applied to search for fainter sources close to the target. The same reduction strategy was used for all filters. For each epoch, the calibration of the S27 mean platescale and the true north orientation was obtained using the $\theta$ Ori $\mathrm{C}$ field observed with HST by McCaughrean \& Stauffer (1994). The same set of stars (TCC058, 057, 054, 034 and 026) were observed with the same observing set-up to avoid introducing any systematic errors (see Table 5 of Chauvin et al. 2010; and Table 2 of Chauvin et al. 2012).

\section{Results}

\subsection{Detection limits}

For each brown dwarf candidate, the $5 \sigma$ detection limit was estimated based on the noise calculated within azimuthal rings of increasing radii centered on the source itself. The noise curve

\footnotetext{
1 http://www.eso.org/projects/aot/eclipse/
}

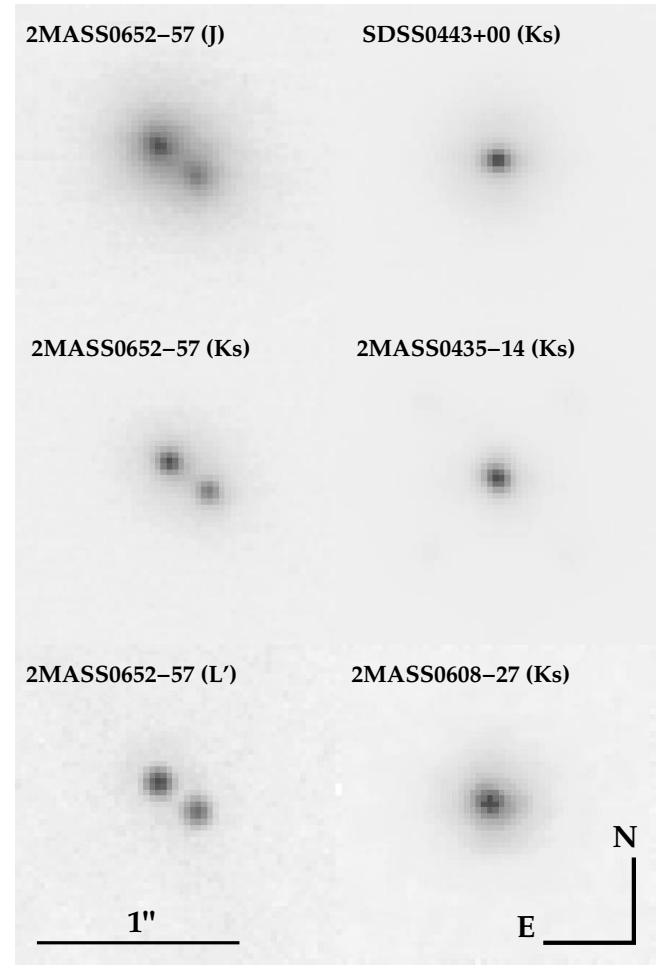

Fig. 1. VLT/NACO image obtained of 2MASS0652-57 in $J, K_{\mathrm{s}}$ and $L^{\prime}$-bands and SDSS0443+00, 2MASS0608-27 and 2MASS0435-14 in $K_{\mathrm{S}}$ band. 2MASS0652-57 is resolved in each band as a close visual binary. All images were normalized to their maximum intensity.

was then divided by the primary star maximum flux. The results are reported for the $K_{\mathrm{s}}$-band filters in Fig. 2, (top). The detection performances vary as a function of both the AO correction (related to the target near-IR brightness and the atmospheric conditions) and the total observing time spent per target to lower the background noise (see Table 2).

Owing to the challenge of observing faint near-IR targets with NACO, the typical angular resolution achieved was about 95 mas, larger than the diffration limit of the telescope in $K_{\mathrm{s}}$ as the typical Strehl correction was lower than $20 \%$. The presence of close companions was therefore probed between $0.1-10^{\prime \prime}$ (i.e. 2-250 AU for a distance of $20 \mathrm{pc}$ ). Based on the DUSTY theoretical models of Chabrier et al. (2000), the detection limits were converted in terms of masses considering both the target near-IR brightness and distance (reported in Table 2) and the ages of 10 and $120 \mathrm{Myr}$, respectively (see Fig. 2, bottom). They are sensitive down to the planetary mass 
Table 3. Relative photometry and astrometry of 2MASS0652-57 AB.

\begin{tabular}{lccccccc}
\hline \hline UT date & $\begin{array}{c}\Delta J \\
(\mathrm{mag})\end{array}$ & $\begin{array}{c}\Delta K_{\mathrm{s}} \\
(\mathrm{mag})\end{array}$ & $\begin{array}{c}\Delta L^{\prime} \\
(\mathrm{mag})\end{array}$ & $\begin{array}{c}\text { Separation } \\
(\mathrm{mas})\end{array}$ & $\begin{array}{c}\text { PA } \\
(\mathrm{deg})\end{array}$ & $\begin{array}{c}\text { Platescale } \\
(\mathrm{mas})\end{array}$ & $\begin{array}{c}\text { True north } \\
(\mathrm{deg})\end{array}$ \\
\hline 2006-01-08 & $0.35 \pm 0.20$ & $0.34 \pm 0.10$ & $0.30 \pm 0.10$ & $228 \pm 6$ & $233.1 \pm 1.7$ & $27.02 \pm 0.06$ & $0.12 \pm 0.13$ \\
2010-08-21 & & $0.31 \pm 0.10$ & & $222 \pm 7$ & $224.1 \pm 1.8$ & $27.02 \pm 0.05$ & $0.0 \pm 0.10$ \\
\hline
\end{tabular}
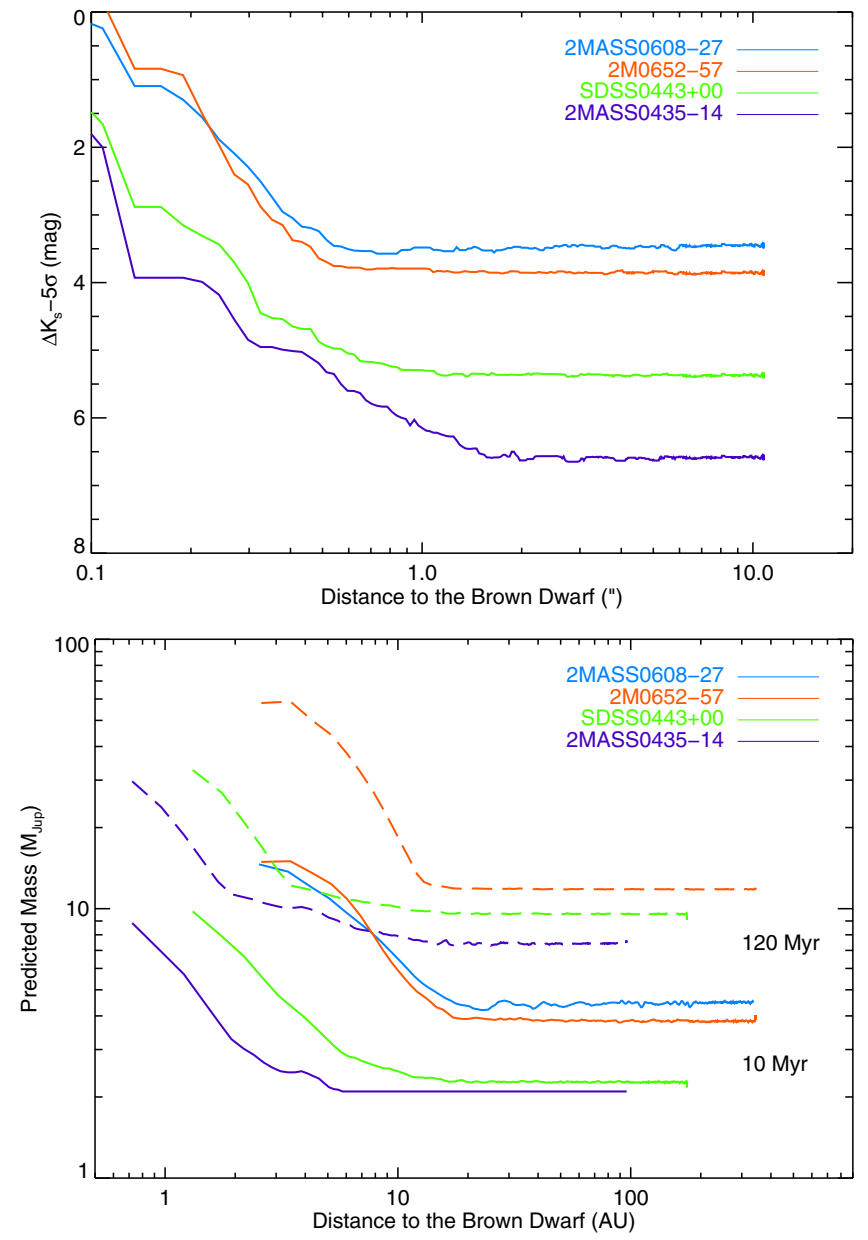

Fig. 2. Top: VLT/NACO detection limits in $K_{\mathrm{s}}$-band of 2MASS0652-57, SDSS0443+00, 2MASS0608-27 and 2MASS0435-14, given here up to $10^{\prime \prime}$. Bottom: detection limits converted in terms of predicted masses as a function of the projected physical separations considering both the DUSTY theoretical model predictions for ages of 10 and $120 \mathrm{Myr}$ and the target properties reported in Table 1. For 2MASS0608-27 probable member of the $\beta$ Pictoris group, only the age of $10 \mathrm{Myr}$ is given. For 2MASS0652-57, part of the image with the binary companion was masked for the detection limit determination.

regime for these ages, but will be degraded to the brown dwarf regime for older ages up to $500 \mathrm{Myr}$. Our best detection limit reaches $2.5 M_{\text {Jup }}$ at $3 \mathrm{AU}$ for $10 \mathrm{Myr}$. The young brown dwarf candidate SDSS0443+00 was observed by Delorme et al. (2012) with NACO in $L^{\prime}$ in December 2009 with deeper detection limits at larger separation (see Fig. 6 of Delorme et al. 2012), but no faint companions were resolved.

\subsection{MASS0652-57, a young brown dwarf binary?}

Among the four young brown dwarf candidates, we discovered that MASS0652-57 was a $\sim 230$ mas visual pair. Both components were resolved in $K_{\mathrm{s}}$, but also in $J$ and $L^{\prime}$-bands (see Fig. 1).

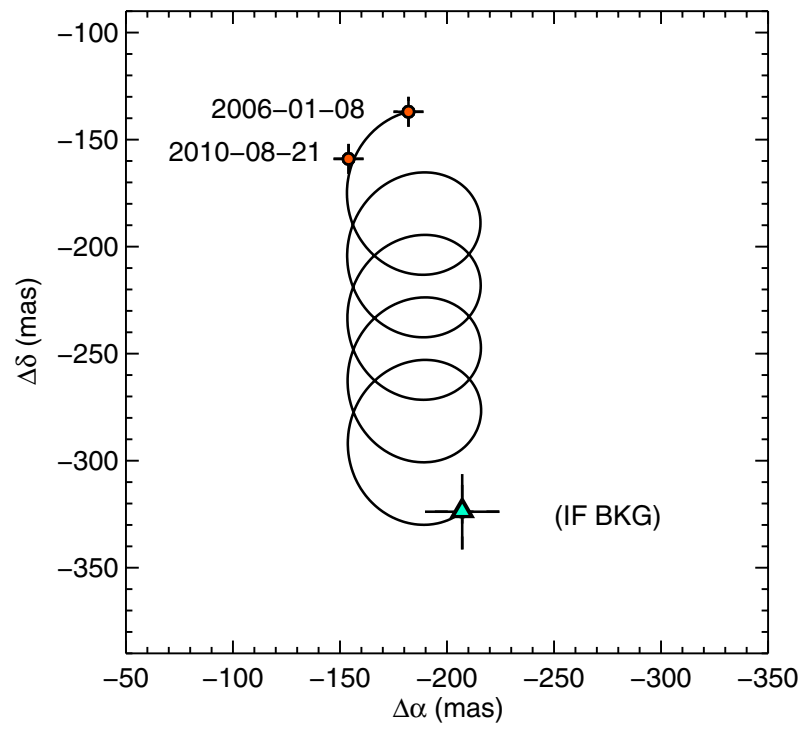

Fig. 3. VLT/NACO measurements with uncertainties in the offset positions of 2MASS0652-57 B relative to A, obtained on January 8, 2006 and August 21, 2010 (circle data points). The solid line gives the expected variation in the offset positions of $B$ relative to $A$, if $B$ is a background stationary object. The triangle data point gives the predicted position for a background object on August 21, 2010. Error bars take into account uncertainties on the initial offset position of B relative to A on January 8, 2006 and on the proper and parallactic motions of 2MASS0652-57 reported by Faherty et al. (2012, see Table 1).

The relative position and photometry between both components were estimated using a classical function fitting (Gaussian and moffat) in all filters for each individual components. The deconvolution algorithm of Véran \& Rigaut (1998) was applied to check the impact of the companion flux contamination. The reported uncertainties are dominated by the dispersion of our results estimated from different image set and related to the fluctuation of the AO correction. The results are given in Table 3. The astrometric results are shown in Fig. 3.

Considering the parallactic and proper motion of 2MASS0652-57 and given the large time span between our observing epochs (4.5 yrs), we can easily reject a stationary background contaminate. We used a $\chi^{2}$ probability test of $2 \times N_{\text {epochs }}$ degrees of freedom following the approach described by Chauvin et al. (2010). We find a probability that the secondary component is a background source lower than $10^{-5}$. We confirm that the system is physically bound as the orbital curvature is unambiguously detected by our two astrometric measurements. This result excludes an edge-on orbital configuration.

Owing to the probability density distribution of the projected physical separation to semi-major axis ratio for a companion to a star (Brandeker et al. 2006), we estimate a probable semimajor axis of $7.3 \pm 3.6 \mathrm{AU}$ for 2MASS0652-57 AB. DUSTY evolutionary models predict a mass of $10-12,15-20,30-40$ and $65-75 M_{\text {Jup }}$ for each component for ages of $10,50,120$ 
and $500 \mathrm{Myr}$, respectively, considering the system's properties reported in Tables 1 and 2. It would imply a $q \sim 0.7-0.8$ mass ratio and a period of 20-200 yrs depending on the system's age. There is a current lack of spectrocopic or kinematics constraints to firmly confirm or reject the youth of this system. 2MASS0652-57 has a well defined parallax and proper motion from Faherty et al. (2012), however it lacks a radial velocity measurement. There is a low-resolution, low SNR optical spectrum available from Cruz et al. (2007), but a significant precise radial velocity can not be extracted from the current data. As such we can not conduct a full UVWXYZ analysis to determine membership in nearby moving groups. Given the proper motion and distance measurement, there is a strong indication of kinematic youth; however without a radial velocity we cannot constrain the age in any significant way. If confirmed as young and if the orbital period remains reasonably short for an astrometric monitoring, the physical properties of the system could provide interesting constraints on evolutionary model predictions over a decade or two (Bonnefoy et al. 2009; Dupuy et al. 2009, 2010; Konopacky et al. 2010).

Acknowledgements. We thank Christine Ducourant, Rama Texeira and Joshua Schlieder for the useful discussion about the status of these young brown dwarf candidates and Alexis Brandeker for his remarks and suggestions as referee of this research note. We want to also thank the staff of ESO-VLT for their support at the telescope. This publication has made use of the SIMBAD and VizieR database operated at CDS, Strasbourg, France. Finally, we acknowledge support from the French National Research Agency (ANR) through project grant ANR10-BLANC0504-01 and the Programmes Nationaux de Planétologie et de Physique Stellaire (PNP \& PNPS), in France.

\section{References}

Ahmic, M., Jayawardhana, R., Brandeker, A., et al. 2007, ApJ, 671, 2074 Béjar, V. J. S., Zapatero Osorio, M. R., Prez-Garrido, A., et al. 2008, A\&A, 477, 895
Biller, B., Allers, K., Liu, M., Close, L. M., \& Dupuy, T. 2011, ApJ, 730, 39 Bonfils, X., Delfosse, X., Udry, S., et al. 2012, A\&A, in press, DOI: $1051 / 0004-6361 / 201014704$

Bonnefoy, M., Chauvin, G., Dumas, C., et al. 2009, A\&A, 506, 799

Bouy, H., Brandner, W., Martín, E. L., et al. 2003, AJ, 126, 1526

Brandeker, A., Jayawardhana, R., Khavari, P., Haisch, K. E. Jr, \& Mardones, D. 2006, ApJ, 652, 1572

Burgasser, A. J., Kirkpatrick, J. D., \& Reid, I. N. 2003, ApJ, 586, 512

Chabrier, G., Baraffe, I., Allard, F., \& Hauschildt, P. H. 2000, ApJ, 542, 464

Chauvin, G., Lagrange, A.-M., Dumas, C., et al. 2004, A\&A, 425, L25

Chauvin, G., Lagrange, A.-M., Bonavita, M., et al. 2010, A\&A, 509, A52

Chauvin, G., Lagrange, A.-M., Beust, H., et al. 2012, A\&A, 542, A41

Cruz, K. L., Reid, I. N., Liebert, J., Kirkpatrick, J. D., \& Lowrance, P. J. 2003, AJ, 126, 2421

Cruz, K. L., Reid, I. N., Kirkpatrick, J. D., et al. 2007, AJ, 133, 439

Delorme, P., Lagrange, A. M., Chauvin, G., et al. 2012, A\&A, 539, A72

Devillar, N. 1997, The messenger, 87

Duchêne, G., Delgado-Donate, E., Haisch, K. E., Jr., Loinard, L., \& Rodríguez, L. F. 2007, PPV (Tucson: University of Arizona Press), 379

Dupuy, T. J., Liu, M. C., \& Bowler, B. P. 2009, ApJ, 706, 328

Dupuy, T. J., Liu, M. C., Bowler, B. P., et al. 2010, ApJ, 721, 1725

Faherty, J. K., Burgasser, A. J., Cruz, K. L., et al. 2009, AJ, 137, 1

Faherty, J. K., Burgasser, A. J., Walter, F. M., et al. 2012, ApJ, 752, 56

Janson, M., Hormuth, F., Bergfors, C., et al. 2012, AJ, submitted

Jayawardhana, R., \& Ivanov, V. D. 2006, Science, 313, 1279

Kirkpatrick, J. D. 2005, ARA\&A, 43, 195

Kirkpatrick, J. D., Barman, T. S., Burgasser, A. J., et al. 2006, ApJ, 639, 1120

Konopacky, Q. M., Ghez, A. M., Barman, T. S., et al. 2010, ApJ, 711, 1087

Luhman, K. H. 2004, ApJ, 602, 816

Marchal, L., Delfosse, X., Forveille, T., et al. 2003, in Brown dwarfs, ed. Martin (San Francisco: Astron. Soc. Pac.), IAU Symp. 211, 311

Mathieu, R. D., Baraffe, I., Simon, M., Stassun, K. G., \& White, R. 2007, PPV (Tucson: University of Arizona Press), 411

Martín, E. L., Brandner, W., Bouvier, J., et al. 2000, ApJ, 543, 299

McCaughrean, M. J., \& Stauffer, J. R. 1994, AJ, 108, 1382

Reid, I. N., Cruz, K.L., Kirkpatrick, J. D., et al. 2008, AJ, 136, 1290

Reiners, A., \& Basri, G. 2009, ApJ, 705, 1416

Rice, E. L., Faherty, J. K., \& Cruz, K. L. 2010, ApJ, 715, 165

Todorov, K., Luhman, K. L., \& McLeod, K. K. 2010, ApJ, 714, 84

Udry, S., \& Santos, N. 2007, ARA\&A, 45, 397

Zapatero Osorio, M. R., Rebolo, R., Martín, E. L., et al. 1998, Brown dwarfs and extrasolar planets, 134,51 